\title{
Variación corporal paralela en peces de dos ríos costeros del Chocó ecuatoriano
}

\section{Pedro Jiménez-Prado ${ }^{1,2 *} \&$ Windsor Aguirre ${ }^{3}$}

1. Pontificia Universidad Católica del Ecuador Sede Esmeraldas, Espejo y Subida a Santa Cruz, 08010065. Esmeraldas, Ecuador; pedro.jimenez@pucese.edu.ec

2. Área de Ecología, Departamento de Ciencias Agrarias y del Medio Natural, Escuela Politécnica Superior de Huesca, Universidad de Zaragoza, Carretera de Cuarte s/n, 22071 Huesca, España.

3. Department of Biological Sciences, DePaul University, 2325 North Clifton Avenue, Chicago, Illinois 60614; waguirre@depaul.edu

* Correspondencia

Recibido 15-V-2020. Corregido 15-IX-2020. Aceptado 02-X-2020.

\begin{abstract}
Parallel body shape variation in fishes from two coastal rivers of the Ecuadorian Chocó. Introduction: Morphological differences are the product of phenotypic plasticity, genetic adaptation or genetic drift, but very old populations are not always required for local adaptation if selective factors are present. Objective: This paper examines the variation in fish body shape in low-altitude coastal rivers to determine if there are common patterns of morphological variation among species and along the course of the watersheds. Methods: From July 2016 to June 2017, a series of ichthyologic samples were collected to analyze the body shape of three species along the entire watercourse of two coastal rivers in Northwestern Ecuador, using geometric morphometrics and multivariate analysis techniques. Results: A parallel change in body shape was found from the lower zone to the upper zone in the three species, characterized by a decrease in body depth. We also found varying levels of allometric variation in the body shape of the three species. Conclusions: Different fish species in these watersheds exhibit parallel changes in body shape along the watercourse that are influenced by ecological and evolutionary processes even though these rivers vary little in altitude, highlighting the importance of these basins as reservoirs of the evolutionary legacy of the fauna and flora in the Neotropics.
\end{abstract}

Key words: morphological parallelism; allometry; geometric morphometrics; Neotropics.

Jiménez-Prado, P., \& Windsor Aguirre, W. (2021). Variación corporal paralela en peces de dos ríos costeros del Chocó ecuatoriano. Revista de Biología Tropical, 69(1), 45-59. DOI 10.15517/rbt.v69i1.41814

La diversidad biológica no se encuentra distribuida de manera homogénea a través del mundo, ni tampoco los factores que la amenazan. Hay áreas con altos niveles de riqueza biológica o endemismo que se encuentran gravemente amenazadas (Myers, Mittermeier, Mittermeier, da Fonseca, \& Kent, 2000). Muchas de estas áreas ya han sido perdidas, mientras que otras están siendo significativamente degradadas (Wilson, 2003; Kolbert, 2015). Lamentablemente, la falta de recursos ha impedido el estudio de la fauna y flora en muchas regiones, resultando en poco conocimiento sobre lo que se está perdiendo y sus consecuencias. Más allá de la reducción de especies, la pérdida de poblaciones genéticamente diferenciadas en ecosistemas separados, ocasionan también la pérdida de porciones significativas del legado evolutivo de esas especies (Moritz, 1994).

La región occidental ecuatoriana es un área con altos niveles de endemismo gracias 
al aislamiento generado por la formación de los Andes hace millones de años (Eigenmann, 1921; Dodson \& Gentry, 1978; Dodson \& Gentry, 1991). Aunque recientemente han existido esfuerzos por obtener información sobre los peces de agua dulce en esta región (e.g., Jiménez-Prado et al., 2015; Valdiviezo-Rivera, Garzón-Santomaro, Inclán-Luna, Mena-Jaén, \& González-Romero, 2018), se conoce poco sobre la mayoría. Aún se describen nuevas especies, pero existen problemas taxonómicos en algunos grupos (Tan \& Armbruster, 2012; Román-Valencia, Ruiz, Taphorn, \& García, 2013; Lujan, Meza-Vargas, \& Barriga-Salazar, 2015; Provezano \& Barriga, 2017); además, hay vacíos de información sobre la ecología de la mayoría de las especies o sobre las diferencias entre poblaciones.

La forma corporal de los peces varía mucho y las diferencias suelen reflejar funciones ecológicas divergentes en el uso del hábitat, como las que se dan entre los peces anádromos y los de agua dulce residentes (Aguirre \& Bell, 2012), entre los arroyos y los lagos (Bolnick et al., 2009) o incluso como respuesta a estresores ambientales (Gharred, Mannai, Belgacem, \& Jebeli, 2020). Estos cambios en la forma del cuerpo se dan por la capacidad de adaptación que puede presentarse entre poblaciones, dentro de la misma cuenca o entre cuencas cercanas geográficamente (Endler, 1977; Bell, 1982; Hendry, Taylor, \& McPhail, 2002; Aguirre, 2009; Aguirre et al., 2016). Además, debido a que suele haber un patrón de cambio similar en las condiciones ambientales entre las partes bajas y altas de los ríos, es posible que también existan patrones de variación morfológica similares entre ríos y especies (Malato et al, 2017; Aguirre et al., 2019).

Específicamente, el noroccidente de la costa ecuatoriana incluye una diversa combinación de cuencas, grandes y pequeñas. Sobre las primeras existe algo de conocimiento (Barriga, 1994; Barriga, 2012; Aguirre et al., 2019), en cambio sobre las pequeñas casi no hay información. Algunos de estos ríos nacen en altitudes por debajo de los $300 \mathrm{msnm}$, desembocan en el mar y son perennes (Fig. 1).
Estos cauces sufren además del impacto de actividades humanas el efecto de especies de peces introducidos (Jiménez-Prado, Vásquez, Rodriguez, \& Taphorn, 2020). Por consiguiente, el objetivo de este estudio fue examinar la variación morfológica de peces en ríos costeros de baja altitud, en los ríos Atacames y Súa al noroccidente ecuatoriano, para determinar si existen patrones de variación morfológica entre especies y entre zonas en estos cauces. Se plantea la hipótesis de que a pesar de que estos ríos son pequeños, las condiciones ambientales entre su desembocadura y su origen, generan diferencias morfológicas intraespecíficas, así como patrones interespecíficos.

\section{MATERIALES Y MÉTODOS}

Área de estudio: El estudio se realizó en los ríos contiguos Atacames y Súa, provincia de Esmeraldas, Ecuador (00 $51^{\prime} 03.9^{\prime \prime} \mathrm{N}$ $79^{\circ} 50^{\prime} 56.2$ "W \& $00^{\circ} 50^{\prime} 32.0$ " $\mathrm{N}-79^{\circ} 53^{\prime} 06.0$ " W). El origen de estos ríos presenta altitudes por debajo de los $200 \mathrm{msnm}$, una extensión del cauce cercana a los $40 \mathrm{~km}$ y un área de drenaje con alrededor de los $300 \mathrm{~km}^{2}$. Estas cuencas son caracterizadas por la presencia de pastos y cultivos en un $79.5 \%$, zona urbana un $3.2 \%$ y parches de bosque natural intervenido en un $17.3 \%$. Esta región se caracteriza por tener una época seca, que va de julio a diciembre, con precipitaciones esporádicas y escasas $(132 \mathrm{~mm})$ y temperatura ambiental promedio de $25.2{ }^{\circ} \mathrm{C}$ (mínima $=20.0$; máxima $=38.0$ ); mientras que de enero a julio se presentan lluvias esporádicas pero abundantes $(459.6 \mathrm{~mm})$ y la temperatura promedio alcanza los $27.1^{\circ} \mathrm{C}$ (mínima $=21.0$; máxima $=37.0)$. A lo largo del gradiente altitudinal, estas cuencas pueden ser subdivididas en tres zonas, según características geomorfológicas, orden del río (Strahler, 1957) y altitud sobre el nivel del mar (Fig. 1). La zona alta del río se caracteriza por presentar mayor pendiente y altitud (colinas altas), lo que forma vertientes de agua con corriente moderada aunque sus cauces son pequeños (orden de río 1 a 2); la zona media del río se caracteriza por tener una menor pendiente y 


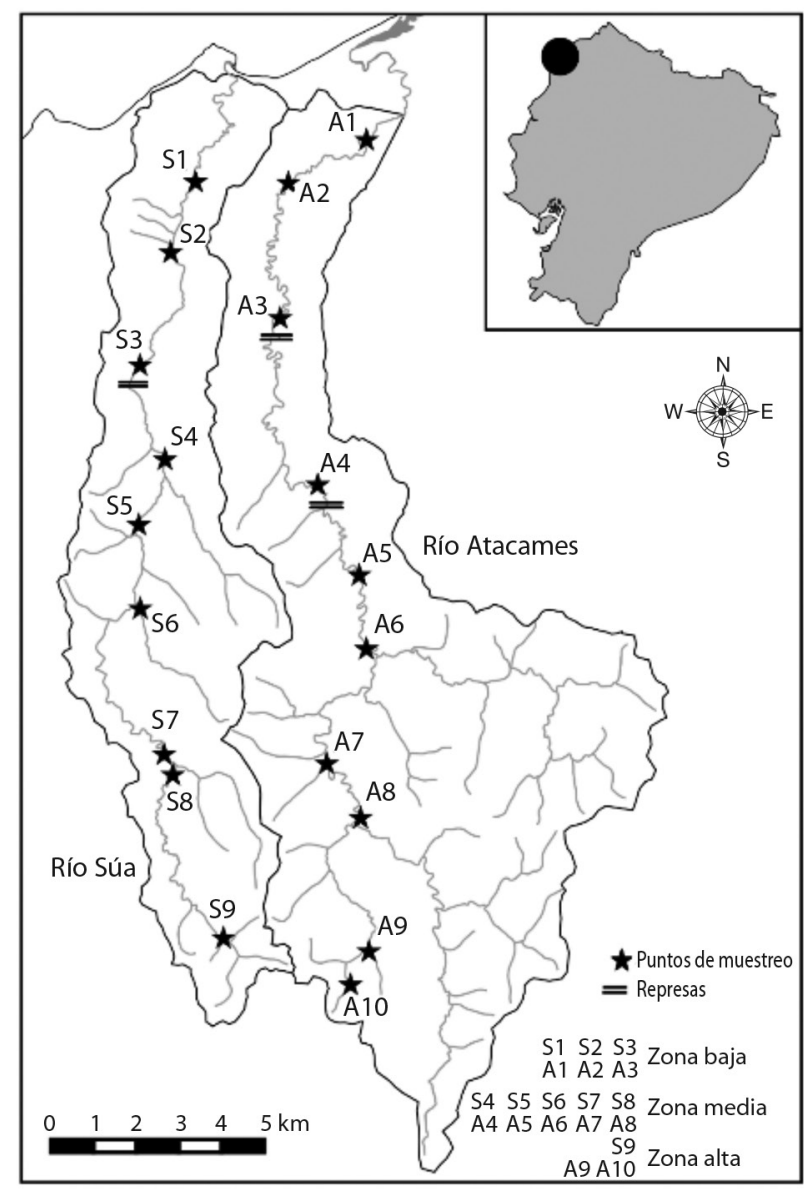

Fig. 1. Área de estudio con localidades de muestreo y separación de zonas de estudio, en la provincia de Esmeraldas, noroccidente de Ecuador.

Fig. 1. Study area with sampling locations and separation of the study zones, province of Esmeraldas, Northwest of Ecuador.

altitud (colinas bajas), lo que forma cauces de agua con corriente moderada y ancho de río moderado (orden de río llega a 4); la zona baja del río se caracteriza por tener una pendiente y altitud leve (llanura), lo que forma cauces de agua con poca corriente y ancho de río moderado a grande (orden de río llega a 6).

Especies analizadas: En estos ríos habitan 15 especies de peces, de las cuales solo tres se encuentran a lo largo de todo el cauce: Rhoadsia minor Eigenmann \& Henn, 1914, Eretmobrycon ecuadorensis Román-Valencia, Ruiz-C, Taphorn, \& García-A 2013 y
Andinoacara blombergi Wijkmark, Kullander \& Barriga Salazar 2012; razón por la cual fueron utilizadas para este estudio. La captura de los peces se realizó entre julio de 2016 a junio de 2017 con muestreos bimensuales a lo largo de los ríos Atacames, en diez localidades distribuidas más o menos de forma equidistante a lo largo del cauce principal y en el río Súa nueve localidades distribuidas de igual forma (Fig. 1).

Los especímenes fueron recolectados con una red de arrastre $(6 \mathrm{~m}$ de largo $\times 1.20 \mathrm{~m}$ de alto y $3 \mathrm{~mm}$ de luz) y una atarraya $(2.0 \mathrm{~m}$ de radio y una luz de $10 \mathrm{~mm}$ ) en tramos de aproximadamente $75 \mathrm{~m}$ de longitud y en un lapso 
de dos horas, siempre con el esfuerzo de dos hombres. Todos los especímenes recolectados se fijaron en una solución de formaldehído al $10 \%$ y se almacenaron durante una semana. En laboratorio los peces fueron identificados taxonómicamente y se conservaron en etanol al $70 \%$ en la colección de peces del museo de la Escuela de Gestión Ambiental (Pontificia Universidad Católica del Ecuador Sede Esmeraldas: CEMZ-p-002-224; 352-368). Cada individuo recolectado fue medido (longitud estándar) con un instrumento de precisión de $0.1 \mathrm{~mm}$ y fue pesado con un instrumento de precisión de $0.01 \mathrm{~g}$. Se analizaron un total de 2544 especímenes, de los cuales 882 fueron de $R$. minor; 1378 de E. ecuadorensis y 284 de A. blombergi.

Toma de datos morfométricos: Se utilizó morfometría geométrica que permiten detectar, cuantificar y visualizar diferencias relativamente pequeñas en la forma del cuerpo, difíciles de detectar usando otros métodos (Toro-Ibacache, Manriquez-Soto \& Suazo-Galdames, 2010; Zelditch, Swiderski \& Sheets, 2012; Benítez \& Püschel, 2014; Aguirre \& Jiménez-Prado, 2018). Cada espécimen utilizado para este análisis fue codificado, según campaña, punto de muestreo e individuo; luego fotografiado con una cámara digital (Nikon D5 $10024 \mathrm{MP}$ con lente AF-S Nikkor 18-55 1:3.5 - 5.6 G), que generó archivos tipo "TPS” en el programa tpsUtil (Rohlf, 2018a), donde se recogieron coordenadas bidimensionales para 16 puntos de referencia (hitos), en $R$. minor y E. ecuadorensis; y 15 puntos de referencia (hitos), en $A$. blombergi. Esta digitalización se realizó para cada espécimen mediante el programa tpsDig2 (Rohlf, 2018b) (Fig. 2).

Análisis de la forma: Las tres especies fueron analizadas por separado en MorphoJ vers. 1.8.0_151 (Klingenberg, 2011). La alineación de Procrustes se realizó por el método de Principal Axes y la matriz de covarianza fue generada para cada especie usando todos los especímenes en conjunto, según cada punto de muestreo donde fueron recolectados y en todo el periodo de estudio. Un análisis de componentes principales (ACP) fue realizado utilizando esta matriz de covarianza para explorar los principales patrones de variación en la forma del cuerpo de cada especie.

El impacto de la alometría fue evaluado mediante una regresión entre el componente principal 1 sobre el logaritmo natural del tamaño centroide (Aguirre \& Jiménez-Prado, 2018). Para diferenciar la forma que adquiere esa alometría, cada especie se organizó en tres grupos, utilizando el rango de los tamaños centroides divididos en tercios iguales de tal manera que el tercio con las tallas menores se la denominó especímenes "pequeños", el segundo tercio como especímenes "medianos" y el tercero como especímenes "grandes". Si el componente de la variación en la forma del cuerpo de cada especie relacionada con la alometría era alto $(\mathrm{P}<0.0001)$ entonces se usaron los residuales de esta regresión, que representan la variación real en la forma del cuerpo independiente al tamaño del espécimen, para hacer análisis de variantes canónicas (AVC) sobre el tamaño centroide. El AVC se realizó para cada especie usando río (Atacames y Súa) y también zona (baja $=$ sitios 1-3, media $=$ sitios 4-8, alta $=$ sitios 9 y 10) por separado como factores para agrupar especímenes.

\section{RESULTADOS}

Los 882 individuos de $R$. minor tuvieron una talla media $=48.9 \mathrm{~mm}$, desviación estándar $(\mathrm{DE})=16.0 \mathrm{~mm}$, mínima $($ Min. $)=19.5 \mathrm{~mm}$, máxima $($ Max. $)=93.3 \mathrm{~mm}$; los 1378 individuos de E. ecuadorensis una talla media $=55.6$ $\mathrm{mm}, \mathrm{DE}=14.6 \mathrm{~mm}$, Min. $=18.9 \mathrm{~mm}$, Max. $=$ $104.9 \mathrm{~mm}$ y los 284 individuos de $A$. blombergi una talla media $=56.5 \mathrm{~mm}, \mathrm{DE}=15.8 \mathrm{~mm}$, Min. $=19.6 \mathrm{~mm}$, Max. $=110.3 \mathrm{~mm}$.

Rhoadsia minor: El ACP realizado indicó fuerte estructura en los datos, en especial en el CP1 (Fig. 3A). CP1 y CP2 explicaron el 48.64 y el $13.39 \%$ de la varianza en la forma del cuerpo, mientras que los demás CPs explicaron el $5.55 \%$ o menos de la variación 


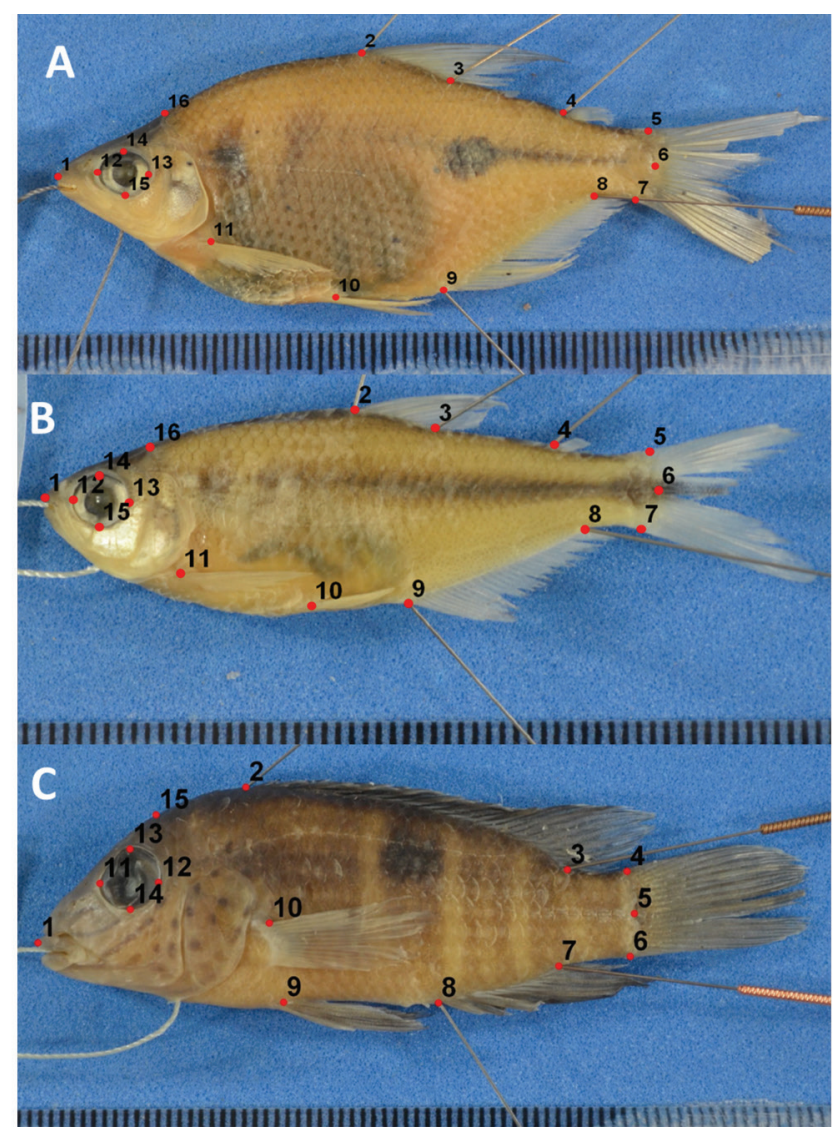

Fig. 2. Especies de estudio e hitos seleccionados para su análisis. A. Rhoadsia minor. B. Eretmobrycon ecuadoriensis. C. Andinoacara blombergi.

Fig. 2. Study species and landmarks selected for the analysis. A. Rhoadsia minor. B. Eretmobrycon ecuadoriensis. C. Andinoacara blombergi.

y fueron descartados. CP1 estuvo muy fuertemente correlacionado con el tamaño de los especímenes $(\mathrm{r}=0.878, \mathrm{~N}=882, \mathrm{P}<0.0001$, Fig. 3B). El análisis de regresión de la forma del cuerpo sobre el tamaño centroide dio un resultado similar, indicando que hubo un fuerte cambio en la forma del cuerpo entre especímenes pequeños y grandes. El tamaño centroide explicó el $38.58 \%$ de la variación del cuerpo en Rhoadsia minor. La alometría resultó en un aumento en la profundidad del cuerpo y una reducción y desplazamiento posterior del ojo en especímenes más grandes (Fig. 3C).

El AVC usando a el río como el factor para determinar grupos dio una buena separación entre especímenes recolectados a través de todo el rango de tamaño de las muestras (Fig. 4A y Fig. 4B), con una distancia de Mahanalobis de 2.413 y distancia de Procrusto de 0.012 entre los promedios de los ríos. Los especímenes de Súa tienen la cabeza un poco más corta y delgada, el ojo más pequeño, el cuerpo más profundo pero el pedúnculo caudal más delgado y la aleta dorsal ligeramente desplazada posteriormente.

El AVC usando la zona de recolección (sitio de baja, media y alta elevación) también indicó que hubo diferencias morfológicas en la altitud de recolección, en especial entre los sitios bajos, los medios y altos (Fig. 5A y Fig. 5B). La variante canónica 1 separó principalmente a especímenes recolectados en sitios bajos de aquellos recolectados en sitios medios 

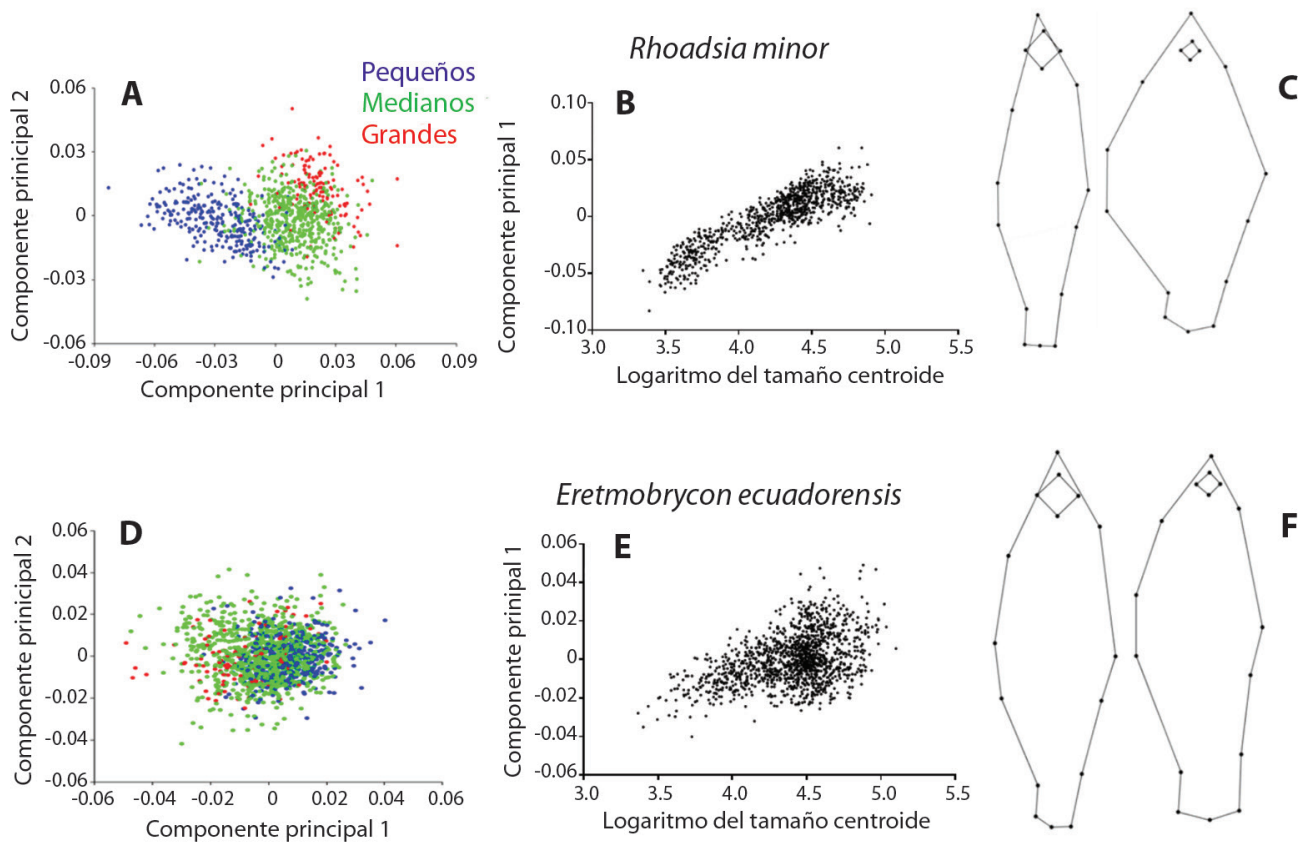

Eretmobrycon ecuadorensis
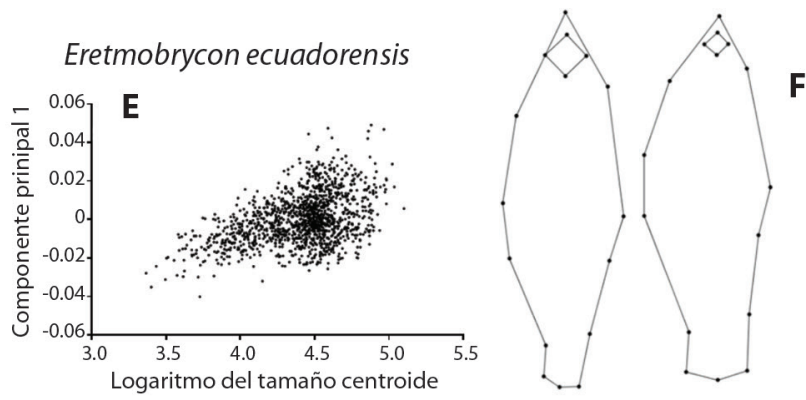

Andinoacara blombergi
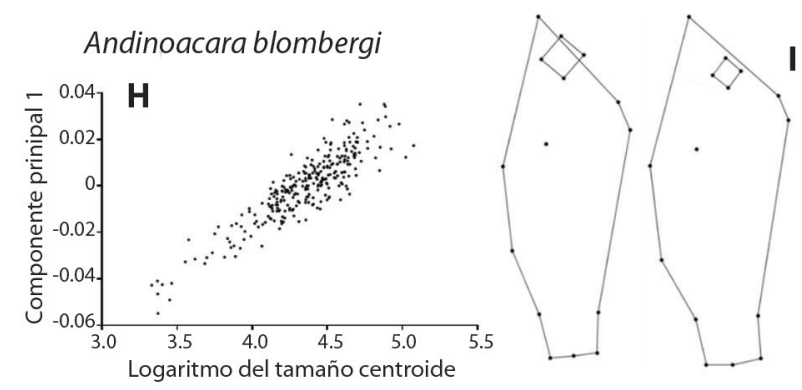

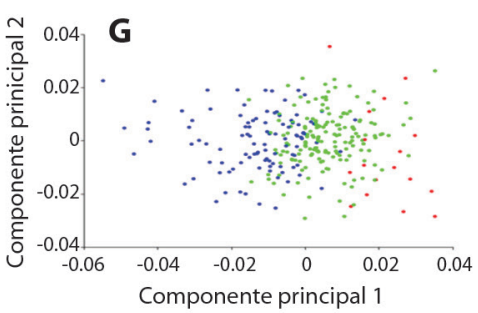

Fig. 3. Relación entre el tamaño y variación en la forma del cuerpo. A, D, G. Análisis de Componentes Principales codificados por su tamaño. B, E, H. Correlación entre el logaritmo del tamaño centroide y el Componente Principal 1. C, F, I. Variación en la forma del cuerpo dado por la alometría. A la izquierda la forma del cuerpo en especímenes pequeños y a la derecha la forma del cuerpo en especímenes grandes, asociado con el Componente principal 1.

Fig. 3. Relationship between size and variation in body shape A, D, G. Principal Components Analysis coded by size. B, E, H. Correlation between the logarithm of centroid size and Principal Component 1. C, F, I. Variation in the shape of the body due to allometry. The body shape of small specimens is on the left and the body shape of large specimens is on the right, as associated with Principal Component 1.

y altos a base de diferencias en la profundidad del cuerpo tanto en la región dorsal como la ventral, mientras que la variante canónica 2 tuvo tendencia a separar a los especímenes recolectados en sitios medios de los sitios altos, a base principalmente de una diferencia en la región abdominal del cuerpo. Como resultado de la diferencia en morfología que se detectó, se realizaron regresiones separadas para cada río de la variación en la forma del cuerpo sobre la altitud del sitio de recolección, ambas fueron significativas y dieron resultados similares en cuanto a la dirección del cambio morfológico.

Eretmobrycon ecuadorensis: El ACP realizado mostró una estructura poco dispersa entre los datos (Fig. 3D). CP1 y CP2 explicaron el 21.35 y el $17.10 \%$ de la varianza en la 

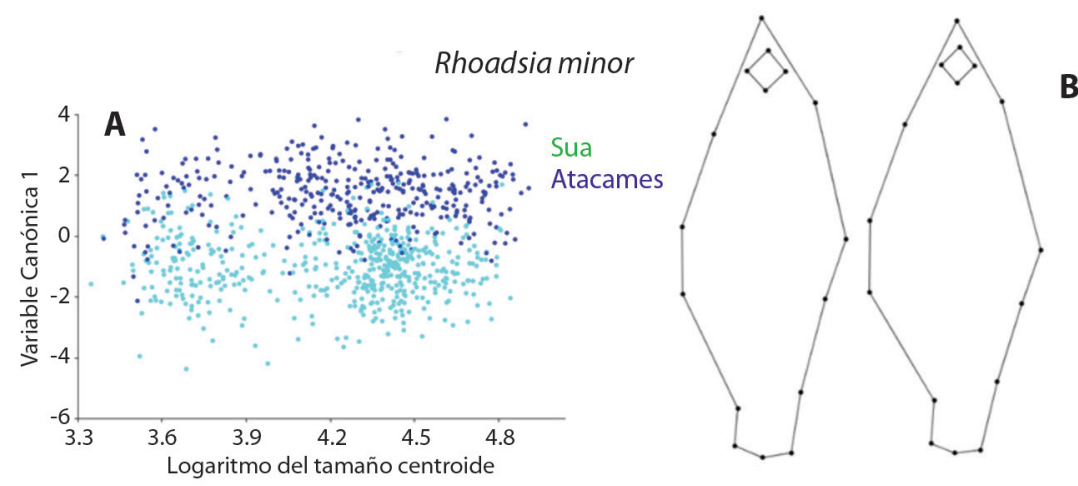

B
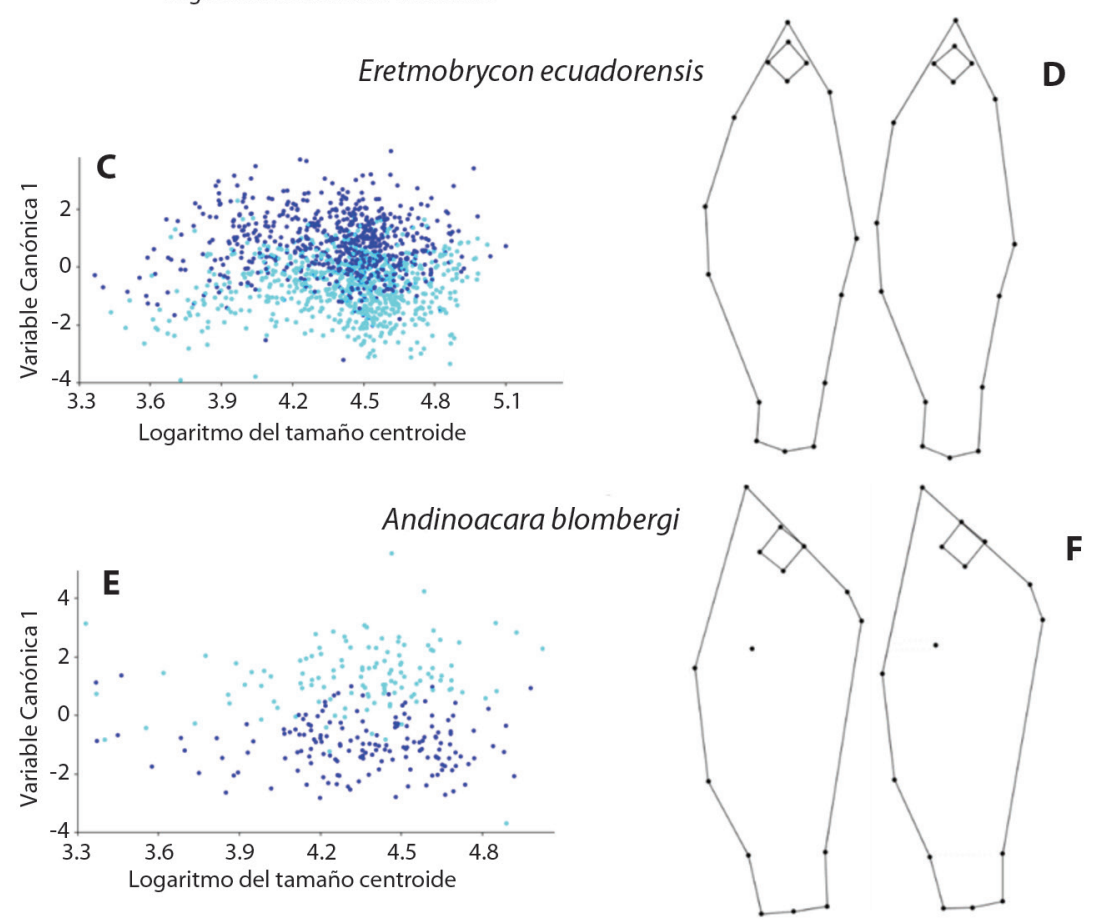

Fig. 4. Forma del cuerpo según el río. A, C, E. Relación entre el logaritmo del tamaño centroide y la Variante Canónica 1. B, D, F. Variación en la forma del cuerpo entre organismos del río Atacames (izquierda) vs. organismos del río Súa (derecha). Fig. 4. Body shape according to the river. A, C, E. Relationship between the logarithm of centroid size and Canonical Variate 1. B, D, F. Variation in body shape between specimens of the Atacames River (left) vs. specimens of the Súa River (right).

forma del cuerpo, los demás CPs explicaron el $13.64 \%$ o menos de la variación y fueron descartados. El CP1 tuvo una correlación moderada con el tamaño de los especímenes $(\mathrm{r}=0.43$, $\mathrm{N}=1378, \mathrm{P}<0.001$, Fig. 3E). El análisis de regresión de la forma del cuerpo sobre el tamaño centroide dio un resultado similar, indicando que hubo poco cambio en la forma del cuerpo entre especímenes pequeños y grandes. El tamaño centroide explicó apenas el $11.41 \%$ de la variación del cuerpo en esta especie. La alometría se manifestó básicamente en una ligera disminución del tamaño del ojo y del alto de la parte supero-frontal de la cabeza (Fig. 3F).

El AVC usando a río como el factor para determinar grupos generó una buena separación entre especímenes recolectados a través de todo el rango de tamaño de las muestras (Fig. 4C y 

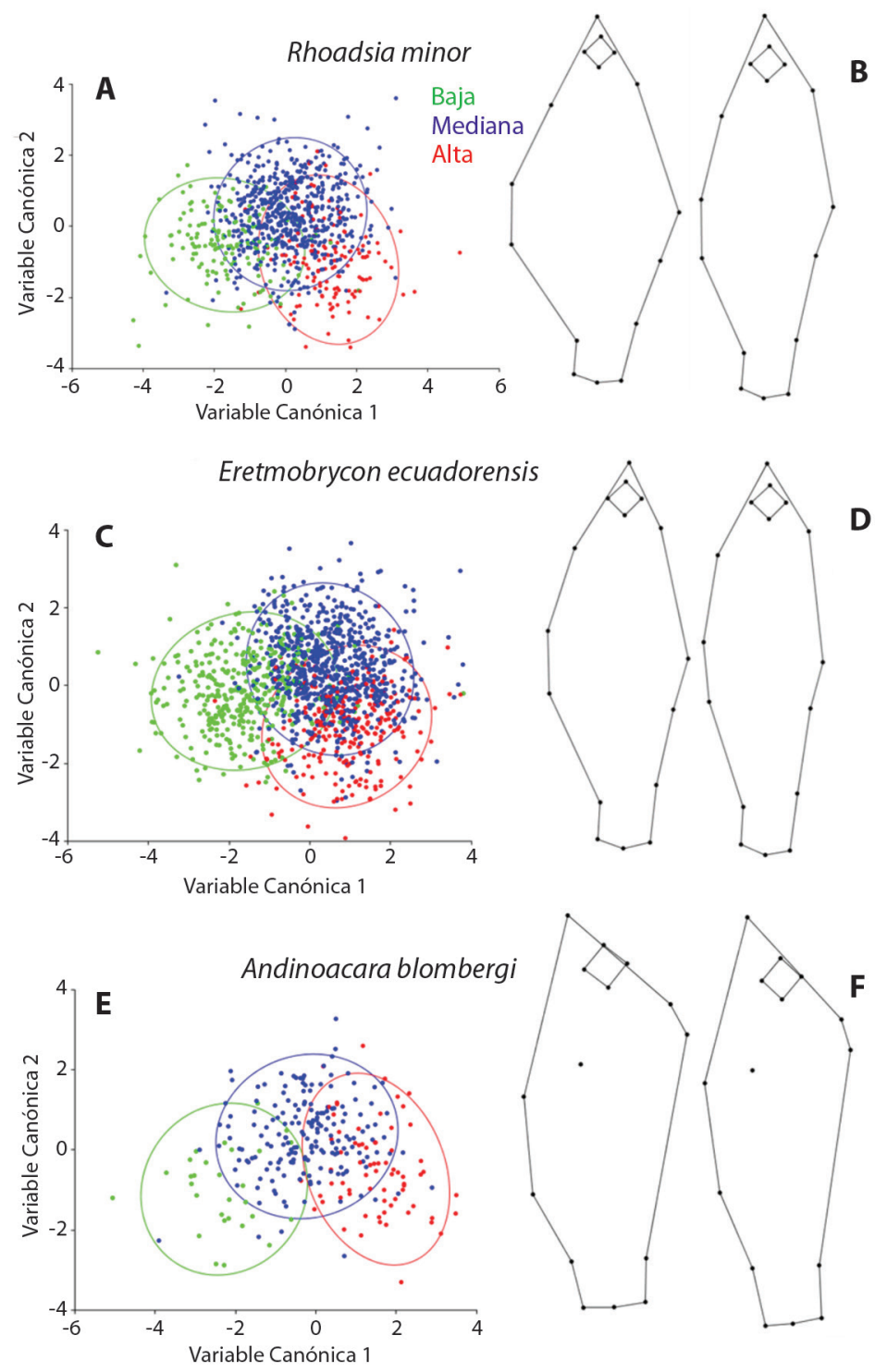

Fig. 5. Relación entre la forma del cuerpo y la zona del río. A, C, E. Análisis de Variante Canónica entre grupos definidos por zona de recolección. B, D, F. Variación en la forma del cuerpo. Organismos de la zona baja (izquierda) vs. organismos de la zona alta (derecha).

Fig. 5. Relationship between the shape of the body and the river zone. A, C, E. Canonical Variate Analysis between groups defined by collection zone. B, D, F. Variation in body shape. Lower zone specimens (left) vs. upper zone specimens (right).

Fig. 4D), con una distancia de Mahanalobis de 1.41 y distancia de Procrusto de 0.0073 entre los promedios de los ríos. El cuerpo de los individuos del río Súa son menos profundos que los del río Atacames, el ojo más pequeño, el hocico algo más pronunciado hacia arriba, así como el inicio de las aletas pélvicas y aleta anal desplazadas hacia atrás, finalmente, la aleta pectoral un poco desplazada hacia abajo.

El AVC usando a las diferentes zonas de recolección (sitio de baja, media y alta elevación) también generó diferencias morfológicas 
con base en la altitud de recolección (Fig. 5C y Fig. 5D). Los patrones que separan a las variantes canónicas 1 y 2 son similares a la especie anterior, en cuanto a que la VC1 separó principalmente a especímenes de sitios bajos de aquellos recolectados en sitios medios y altos; dado principalmente por una diferencia en el alto del cuerpo, tanto en la región dorsal como la ventral; algo parecido sucede en la VC2 donde también se presenta una tendencia a separar a los especímenes recolectados en sitios medios de los sitios altos, dado principalmente por una diferencia en el tamaño del ojo. En las regresiones separadas para cada río, también dieron resultados similares en cuanto a la dirección del cambio morfológico y la altitud.

Andinoacara blombergi: El ACP realizado mostró una estructura muy dispersa entre los datos, en particular en el CP1 (Fig. 3G). CP1 y CP2 explicaron el 26.43 y el $14.53 \%$ de la varianza en la forma del cuerpo, los demás CPs explicaron el $12.69 \%$ o menos de la variación $\mathrm{y}$ fueron descartados. El CP1 tuvo una fuerte correlación con el tamaño de los especímenes $(\mathrm{r}=0.89, \mathrm{~N}=284, \mathrm{P}<0.001$, Fig. 3H). El análisis de regresión de la forma del cuerpo sobre el tamaño centroide dio un resultado similar, indicando que hubo un cambio moderado en la forma del cuerpo entre especímenes pequeños y grandes. El tamaño centroide explicó el $21.78 \%$ de la variación del cuerpo en esta especie. La alometría se manifestó en una disminución del tamaño del ojo, un ligero aumento en el alto del cuerpo a nivel de la cabeza, tanto en la parte dorsal como ventral, y en un pequeño desplazamiento hacia abajo de la aleta pectoral (Fig. 3I).

El AVC usando a los ríos como factor que determina los grupos, también generó una buena separación entre especímenes recolectados a través de todo el rango de tamaño de las muestras (Fig. 4E y Fig. 4F), con una distancia de Mahanalobis de 2.31 y distancia de Procrusto de 0.011 entre los promedios de los ríos. $\mathrm{La}$ cabeza de los individuos de río Súa presenta el ojo más grande y un hocico algo más pronunciado que la cabeza de los individuos del río
Atacames, así como un pedúnculo caudal algo más delgado.

Para esta especie, el AVC usando la zona de recolección (sitio de baja, media y alta elevación) también generó diferencias morfológicas en la altitud de recolección (Fig. 5E y Fig. 5F). Los patrones que separan a las variantes canónicas 1 y 2 son similares a las especies anteriores, en cuanto a que la $\mathrm{VC} 1$ separó principalmente a individuos de sitios bajos de aquellos recolectados en sitios medios y altos; dado principalmente por una diferencia en el alto del cuerpo, tanto en la región dorsal como la ventral, por detrás de la cabeza; algo parecido sucede también en la VC2 donde se presenta una tendencia a separar a los especímenes recolectados en sitios medios de los sitios altos y bajos, dado principalmente por una diferencia en el tamaño del ojo. En las regresiones separadas para cada río, también dieron resultados similares en cuanto a la dirección del cambio morfológico y la altitud.

\section{DISCUSIÓN}

Con el presente estudio, se ha demostrado, primero que hay diferencias en la forma del cuerpo entre poblaciones de las dos cuencas a pesar de que estas están geográficamente adyacentes, con tamaño, gradiente y condiciones ambientales similares; es decir, esto resalta la influencia del aislamiento geográfico como un factor que impulsa la variación fenotípica de los peces. Segundo, existe un patrón paralelo de diferenciación morfológica para las tres especies en ambas cuencas muestreadas: la profundidad del cuerpo fue mayor en las partes bajas de la cuenca que en las partes altas, resaltando aún más la capacidad de los peces de adaptarse a condiciones locales incluso dentro de la misma cuenca geográfica. Tercero, se encontró que un componente significativo de la variación en la forma del cuerpo en las tres especies está asociado con la alometría, el cambio en la forma asociado con el desarrollo.

En cuanto a la variación fenotípica microgeográfica, hay que señalar que la diversidad de peces por unidad de área es mucho mayor en 
sistemas de agua dulce que en el mar (Nelson, Grande, \& Wilson, 2016). Esto se debe, por lo menos en parte, a la mayor abundancia de barreras geográficas que existen en sistemas de agua dulce y las resultantes oportunidades para la diferenciación genética y la adaptación local. Diferencias ambientales en el hábitat también pueden resultar en diferencias morfológicas entre poblaciones mediante la plasticidad fenotípica (West-Eberhard, 2003). Como consecuencia, la variación fenotípica es relativamente común entre poblaciones geográficamente aisladas de peces de agua dulce (Sidlauskas, Chernoff \& Machado-Allison, 2006; Aguirre, 2009; Fonseca de Barros, Louvise \& Pellegrini-Caramaschi, 2019).

El análisis de variantes canónicas separando poblaciones por río en este estudio mostró que hubo diferencias detectables en la forma del cuerpo entre poblaciones en los ríos Atacames y Súa para las tres especies muestreadas a través del rango de tamaños para cada especie (Fig. 4). Sin embargo, la magnitud de la diferencia entre ríos fue relativamente pequeña y el patrón de diferenciación no fue consistente entre las especies; cada especie mostró su propio patrón de divergencia morfológica entre ríos. Diferencias pequeñas en la forma del cuerpo como estas pueden ser el producto de muchos factores incluyendo procesos aleatorios. Se necesitaría muestrear más ríos para poder identificar los factores importantes que causan la divergencia morfológica entre poblaciones habitando diferentes cuencas hidrográficas en la costa ecuatoriana y estimar su magnitud típica.

El patrón de diferenciación más interesante fue el que se detectó entre poblaciones habitando las partes bajas y altas de las dos cuencas. En las tres especies y en ambos ríos, las poblaciones muestreadas en las partes bajas de la cuenca tuvieron el cuerpo más profundo que las que habitan las partes altas (Fig. 5). Este tipo de cambio en la forma del cuerpo ha sido reportado previamente en varias especies incluyendo al género Rhoadsia (Sidlauskas, Chernoff, \& Machado-Allison, 2006; Aguirre et al., 2016; Malato et al., 2017; Aguirre et al., 2019). En todos los ríos en que se ha examinado la variación en la forma del cuerpo entre poblaciones de Rhoadsia, la profundidad relativa del cuerpo disminuye con aumento en la elevación, en especial en tramos cuya altitud varía de los 500 a $1000 \mathrm{~m}$. Mientras que las cuencas de los ríos Atacames y Súa que son mucho más pequeñas y de menor cambio altitudinal (entre $5 \mathrm{y}$ $130 \mathrm{~m}$ ), se ha reportado también la divergencia morfológica para Rhoadsia y es la primera vez que se ha documentado, este tipo de cambios en la profundidad del cuerpo con la altitud del río para E. ecuadorensis y A. blombergi.

Hay varios factores que cambian en paralelo con la elevación de los ríos Neotropicales que podrían ser responsables individualmente o en conjunto de este patrón común de divergencia en la forma del cuerpo (Vannote, Minshall, Cummins, Sedell, \& Cushing, 1980; Sidlauskas et al., 2006; Winemiller, Agostinho, \& Caramaschi, 2008; Jaramillo-Villa, Maldonado-Ocampo, \& Escobar, 2010; Aguirre et al., 2016; De la Barra, Zubieta, Aguilera, Maldonado, Pouilly, \& Oberdorff, 2016). Por ejemplo, las partes bajas de las cuencas suelen tener una proporción más alta de hábitats con aguas lentas o quietas, el agua suele ser más caliente, los peces ictiófagos son más abundantes y los nutrientes se acumulan. Por otra parte, en las partes altas los ríos son más angostos, con rocas entre las cuales fluye el agua con mayor velocidad, la temperatura suele ser más baja, los peces ictiófagos son menos abundantes o están ausentes y los nutrientes son más escasos (Vannote et al., 1980; Winemiller et al., 2008; Aguirre et al., 2016, Aguirre et al., 2019). De estos factores, es ampliamente conocido que un cuerpo más hidrodinámico (menos profundo) en peces está fuertemente asociado con hábitats de mayor flujo por razones de eficiencia energética (Webb, 1984; Langerhans \& Reznick, 2010).

Aspectos bióticos como la abundancia de depredadores y alimentos podrían también estar actuando. Una mayor profundidad del cuerpo en peces funciona como una defensa en contra de peces ictiófagos de manera que poblaciones sujetas a fuerte depredación por peces suelen 
tener el cuerpo más profundo (Andersson, Johansson, \& Soderlund, 2006; Chivers, Zhao, Brown, Marchant, \& Ferrari, 2008). También es común que poblaciones de peces que habitan ambientes ricos en nutrientes y alimentos suelen tener cuerpos más profundos y robustos (Andersson et al., 2006; Chivers et al., 2008). Incluso diferencias pequeñas en temperatura pueden causar cambios en la forma del cuerpo (Reyes-Corral \& Aguirre, 2019). Futuros estudios deberían tomar variables ambientales específicas, en este tipo de cuencas, para diseñar experimentos que cuantifiquen como están contribuyendo los diferentes factores a la variación morfológica que hemos documentado.

No está claro si las diferencias morfológicas documentadas son el producto de la plasticidad fenotípica, diferencias genéticas producto de la adaptación local o la deriva genética, o una combinación de estas. Los elementos de cambio en la distribución del gradiente, diferencias en el flujo del agua, la temperatura, la alimentación o la presencia de depredadores, en cuanto al cambio en la forma del cuerpo evidenciados, podrían estar relacionados con una respuesta adaptativa producto de la plasticidad fenotípica ante la presencia de agentes externos (Fisk et al., 2007). La plasticidad que es común en los peces y que pueden influenciar en el desarrollo del cuerpo y resultar en diferencias morfológicas entre poblaciones que no requieren cambios genéticos (West-Eberhard, 2003; Andersson et al., 2006; Chivers et al., 2008; Reyes-Corral \& Aguirre, 2019). Estudios actuales muestran que la capacidad de las poblaciones para experimentar una evolución contemporánea o rápida es también posible (Swanne, Hendry, \& Reznick, 2017), donde la plasticidad fenotípica puede influir en la dinámica evolutiva $\mathrm{y}$, por lo tanto, en las tasas de evolución.

Por otro lado, la adaptación genética también es común en peces de río y es posible que haya un componente de adaptación genética local que esté influenciando las diferencias en la forma del cuerpo entre poblaciones en las partes bajas y altas de estos ríos (Hendry et al., 2002). Las poblaciones ni si quiera requieren ser muy antiguas para estar adaptadas localmente si hay diferencias fuertes en los factores selectivos; además la evolución contemporánea puede resultar en cambios genéticos y fenotípicos en pocas generaciones (Hendry \& Kinnison, 1999; Aguirre \& Bell, 2012). Se ha demostrado incluso que caracteres como el tamaño corporal y la forma del cuerpo son evolutivamente más probables de un cambio temprano y en espacios cercanos (Blomberg, Garland, \& Ives, 2003).

En cuanto a la alometría, hay que entender que los estudios sobre la importancia de la variación morfológica asociada con el desarrollo y la alometría tienen una larga historia en la biología (Thompson, 1917; Huxley, 1932; Gould, 1977). Por ello, en la actualidad, es la morfometría geométrica una de las metodologías más usadas como una poderosa herramienta para este propósito (Rohlf \& Marcus, 1993; Zelditch et al., 2012; Klingenberg, 2016).

En este estudio, un componente significativo de la variación intraespecífica en la forma del cuerpo de las tres especies examinadas estuvo asociada con la alometría. Este factor toma importancia cuando se presentan rangos amplios de tamaños, pero también la alometría en los peces es común (Friedman, Martinez, Price, \& Wainwright, 2019). Es importante ver la magnitud en el porcentaje de la varianza en la forma del cuerpo explicada por la alometría que difirió sustancialmente entre especies. La alometría fue muy fuerte en $R$. minor explicando el $38.58 \%$ de la varianza en la forma del cuerpo; con grado intermedio en A. blombergi, que explicó el $21.78 \%$ de la varianza, y finalmente E. ecuadorensis, que explicó solo el $11.41 \%$ de la varianza.

Rhoadsia minor ha mostrado diferenciación entre poblaciones tanto en este estudio (Fig. 4 y Fig. 5) como en estudios previos junto a su congénere $R$. altipinna (Aguirre et al., 2016; Malato et al. 2017). La manera en que cambia la forma del cuerpo con el desarrollo también coincide con la manera más obvia en que difiere la forma del cuerpo entre poblaciones de Rhoadsia; ambas involucran cambios en la profundidad relativa del cuerpo. 
El cuerpo de ejemplares pequeños de Rhoadsia es relativamente similar en profundidad al de otros carácidos en la región occidental del Ecuador. A medida que crecen, hay un crecimiento alométrico positivo importante en la profundidad del cuerpo de tal manera que los adultos desarrollan cuerpos que están entre los más profundos de la familia Characidae en el Ecuador occidental (Jiménez-Prado et al., 2015; Aguirre et al., 2016).

Sería importante investigar más detalladamente si la alometría relativamente fuerte en la forma del cuerpo, que parece caracterizar a especies del género Rhoadsia, puede servir como una fuente de variación morfológica y facilitar el proceso de diferenciación entre poblaciones, así como su relación con las características del hábitat. Esto es porque en teoría debería ser más fácil cambiar programas de desarrollo que ya existen que producir programas de desarrollo nuevos. Dado que parte normal del programa de desarrollo de Rhoadsia incluye un cambio relativamente fuerte en la forma del cuerpo, de menos profundo a más profundo a medida que los individuos crecen, la evolución de las poblaciones con cuerpos menos profundos que generalmente se encuentran en las partes más altas de las cuencas simplemente involucraría reducir la tasa de crecimiento del cuerpo en el eje ventral-dorsal relativo al programa normal de desarrollo. Es decir, cambiar el crecimiento alométrico positivo en la profundidad del cuerpo característico de las poblaciones en las partes bajas a un crecimiento más isométrico. En este sentido, Sidlauskas et al. (2006) reportaron que diferencias en las trayectorias de crecimiento alométrico contribuyen a la diferencia en la profundidad del cuerpo entre poblaciones de Bryconops $s p$. cf. melanurus habitando las partes bajas y altas de la región del Pantanal Brasileño. Estudios comparados de la alometría en otros grupos nos han enseñado mucho acerca de cómo y cuándo en el desarrollo surgen las diferencias morfológicas entre especies y podrían ser de provecho aquí (Zelditch et al., 2012; Klingenberg, 2016).

Los resultados de este estudio indican que aún en las cuencas hidrográficas pequeñas, existen patrones de variación morfológica importante que sugieren procesos ecológicos y evolutivos influenciando la variación fenotípica de los peces de diferente manera en diferentes partes de las cuencas. Dado que estas cuencas pequeñas son altamente vulnerables a la degradación ambiental, esto es una causa para reflexionar sobre lo que se está perdiendo a medida que las condiciones ambientales en estos ecosistemas se deterioran y especies se extinguen localmente. En muchos casos, es probable que poblaciones localmente adaptadas o incluso especies no descritas ya se hayan extinguido sin nunca haber sido estudiadas en detalle.

Este es el estudio de variación morfológica de peces más detallado que se ha realizado hasta la fecha en las cuencas pequeñas de la costa noroccidental del Ecuador, y resalta la importancia que tienen estas cuencas como reservorios del legado evolutivo de la fauna y flora neotropical. Esperamos que este estudio estimule más investigación a nivel poblacional sobre la variación fenotípica y genética en peces de la región.

Declaración de ética: los autores declaran que todos están de acuerdo con esta publicación y que han hecho aportes que justifican su autoría; que no hay conflicto de interés de ningún tipo; y que han cumplido con todos los requisitos y procedimientos éticos y legales pertinentes. Todas las fuentes de financiamiento se detallan plena y claramente en la sección de agradecimientos. El respectivo documento legal firmado se encuentra en los archivos de la revista.

\section{AGRADECIMIENTOS}

Este trabajo es resultado de los proyectos con fondos internos de investigación de la Pontificia Universidad Católica del Ecuador Sede en Esmeraldas de los años 2016 y 2018; Los resultados de este trabajo forman parte de la tesis del primer autor dentro del Doctorado en Ciencias Agrarias y del Medio Natural de la Universidad de Zaragoza, con el apoyo del 
Banco Santander; agradecemos la colaboración Fernando Vásquez en los muestreos de campo y Michel Mora por la digitalización de imágenes.

\section{RESUMEN}

Introducción: Las diferencias morfológicas son el producto de la plasticidad fenotípica, la adaptación genética o la deriva genética, pero no siempre se requiere de poblaciones muy antiguas para conseguir adaptaciones locales si se presentan factores selectivos. Objetivo: Este trabajo examina la variación en la forma del cuerpo de peces en ríos costeros de baja altitud para determinar si existen patrones de variación morfológica entre especies y entre zonas a lo largo de estos cauces. Métodos: Desde julio 2016 a junio 2017 se realizaron una serie de muestreos ictiológicos que permitieron analizar la forma del cuerpo de tres especies, a lo largo de todo el cauce en dos ríos costeros del noroccidente ecuatoriano, con el uso de la morfometría geométrica y técnicas de análisis multivariante. Resultados: Se encontró un paralelismo en el cambio de la forma del cuerpo de las tres especies, desde la zona baja hacia la zona alta, caracterizado por una reducción en la profundidad del cuerpo. También encontramos diferentes niveles de variación alométrica en la forma del cuerpo de las tres especies. Conclusiones: Peces en estas cuencas presentan variación fenotípica influenciada por procesos ecológicos y evolutivos que se expresan en cambios paralelos en la forma del cuerpo en diferentes especies incluso en ríos que tienen rangos de altitud muy pequeños, resaltando la importancia que tienen estas cuencas como reservorios del legado evolutivo de la fauna y flora neotropical.

Palabras clave: paralelismo morfológico; alometría; morfometría geométrica; Ecuador noroccidental; Neotrópico.

\section{REFERENCIAS}

Aguirre, W.E., \& Bell, M.A. (2012). Twenty years of body shape evolution in a threespine stickleback population adapting to a lake environment. Biological Journal of the Linnean Society, 105, 817-831.

Aguirre, W., \& Jiménez-Prado, P. (2018). Guía práctica de morfometría geométrica. Aplicaciones en la ictiología. Esmeraldas, Ecuador: Pontificia Universidad Católica del Ecuador Sede Esmeraldas (PUCESE).

Aguirre, W.E. (2009). Microgeographical diversification of threespine stickleback: body shape-habitat correlations in a small, ecologically diverse Alaskan drainage. Biological Journal of the Linnean Society, 98, 139-151.

Aguirre, W.E., Navarrete, R., Malato, G., Calle, P., Loh, M.K., Vital, W.F., ... Granda, J.C. (2016). Body
Shape variation and population genetic structure of Rhoadsia altipinna (Characidae: Rhoadsiinae) in Southwestern Ecuador. Copeia, 104, 554-569.

Aguirre, W.E., Young, A., Navarrete-Amaya, R., Valdiviezo-Rivera, J., Jiménez-Prado, P., Cucalón-Tamayo, R., ... Shervette, V. (2019). Vertebral number covaries with body form and elevation along the Western Slopes of the Ecuadorian Andes in the Neotropical fish genus Rhoadsia (Teleostei: Characidae). Biological Journal of the Linnean Society, 126, 706-720.

Andersson, J., Johansson, F., \& Soderlund, T. (2006) Interactions between predator- and diet-induced phenotypic changes in body shape in crucian carp. Royal Society of London, 273, 431-437.

Barriga, R. (2012). Lista de peces de agua dulce e intermareales del Ecuador. Revista Politécnica, 30, 83-119.

Barriga, R. (1994). Peces del Noroeste del Ecuador. Revista Politécnica, 19, 43-154.

Bell, M.A. (1982). Differentiation of adjacent stream populations of threespine sticklebacks. Evolution, $36,189-199$.

Benítez, H.A., \& Püschel, T.A. (2014). Modelando la varianza de la forma: Morfometría geométrica aplicaciones en biología evolutiva. International Journal of Morphology, 32, 998-1008.

Blomberg, S., Garland, T., \& Ives, A. (2003). Testing for phylogenetic signal in comparative data: behavioral traits are more labile. Evolution, 57, 717-745.

Bolnick, D.I., Snowberg, L., Patenia, C., Lau, O.L., Stutz, W.E., \& Ingram, T. (2009). Habitat choice contributes to adaptive divergence between lake and stream populations of threespine stickleback. Evolution, 63, 2004-2016.

Chivers, D.P., Zhao, X., Brown, G.E., Marchant, T.A., \& Ferrari, M.C.O. (2008). Predator-induced changes in morphology of a prey fish: the effects of food level and temporal frequency of predation risk. Evolutionary Ecology, 22, 561-574.

De la Barra, E., Zubieta, J., Aguilera, G., Maldonado, M., Pouilly, M., \& Oberdorff, T. (2016). ¿Qué factores determinan la distribución altitudinal de los peces de ríos tropicales andinos? Revista de Biología Tropical, 64, 157-176.

Dodson, C.H., \& Gentry, A.H. (1978). Flora of the Rio Palenque Science Center, Selbyana. The Journal of the Marie Selby Botanical Gardens, 4, 1-628.

Dodson, C.H., \& Gentry, A.H. (1991). Biological Extinction in Western Ecuador. Annals of the Missouri Botanical Garden, 78, 273-295.

Eigenmann, C.H. (1921). The nature and origin of the fishes of the Pacific slope of Ecuador, Peru and Chili. 
Proceedings of the American Philosophical Society, 51, 503-523.

Endler, J.A. (1977). Geographic variation, speciation, and clines. New Jersey, USA: Princeton University Press.

Fisk, D.F., Latta, L.C., Knapp, R.A., \& Pfrender, M.E. (2007). Rapid evolution in response to introduced predators I: rates and patterns of morphological and life-history trait divergence. BMC Evolutionary Biology, 7, 22.

Fonseca de Barros, T., Louvise, J., \& Pellegrini-Caramaschi, E. (2019). Flow gradient drives morphological divergence in an Amazon pelagic stream fish. Hydrobiologia, 833, 217-229.

Friedman, S.T., Martinez, C.M., Price, S.A., \& Wainwright, P.C. (2019). The influence of size on body shape diversification across Indo-Pacific shore fishes. Evolution, 73, 1873-1884.

Gharred, T., Mannai, R., Belgacem, M., \& Jebeli, J. (2020). Incidence of morphometry variation, growth alteration, and reproduction performance of the annular sea bream (Diplodus annularis) as effective tools to assess marine contamination: how useful is a multi-biotimarkers approach? Environmental Science and Pollution Research, 27, 4075-4088.

Gould, S.J. (1977). Ontogeny and phylogeny. Cambridge, USA: Belknap Press of Harvard University Press.

Hendry, A.P., \& Kinnison, M.T. (1999). The pace of modern life: measuring rates of contemporary microevolution. Evolution, 53, 1637-1653.

Hendry, A.P., Taylor, E.B., \& McPhail, J.D. (2002). Adaptive divergence and the balance between selection and gene flow: lake and stream stickleback in the Misty system. Evolution, 56, 1199-1216.

Huxley, J.S. (1932). Problems of Relative Growth. Lincoln Lac Veagh. New York, USA: The Dial Press.

Jaramillo-Villa, U., Maldonado-Ocampo, J.A., \& Escobar, F. (2010). Altitudinal variation in fish assemblage diversity in streams of the central Andes of Colombia. Journal of Fish Biology, 76, 2401-2417.

Jiménez-Prado, P., Aguirre, W., Laaz-Moncayo, E., Navarrete-Amaya, R., Nugra-Salazar, F., Rebolledo-Monsalve, E., ... Valdiviezo-Rivera, J. (2015). Guía de Peces para aguas continentales en la vertiente occidental del Ecuador. Esmeraldas, Ecuador: Pontificia Universidad Católica del Ecuador Sede Esmeraldas (PUCESE); Universidad del Azuay (UDA) y Museo Ecuatoriano de Ciencias Naturales (MECN) del Instituto de Biodiversidad.

Jiménez-Prado, P., Vásquez, F., Rodríguez, D., \& Taphorn, D. (2020). Efectos de la especie invasora Poecilia gillii (Cyprinodontiformes: Poecilidae) sobre Pseudopoecilia fria en de los ríos costeros de la región del Chocó, Ecuador. Revista de Biología Tropical, $68,122-138$.

Klingenberg, C.P. (2011). MorphoJ: an integrated software package for geometric morphometrics. Molecular Ecology Resources, 11, 353-357.

Klingenberg, C.P. (2016). Size, shape, and form: concepts of allometry in geometric morphometrics. Development Genes and Evolution, 226, 113-137.

Kolbert, E. (2015). The sixth extinction. An unnatural history. London, UK: Picador.

Langerhans, R.B., \& Reznick, D.N. (2010). Ecology and evolution of swimming performance in fishes: predicting evolution with biomechanics. En P. Domenici, \& B.G. Kapoor (Eds.), Fish Locomotion: An Ethoecological Perspective (pp. 200-248). Enfield, New Hampshire, UK: Science Publishers.

Lujan, N.K., Meza-Vargas, V., \& Barriga-Salazar, R. (2015). Two New Chaetostoma Group (Loricariidae: Hypostominae) Sister Genera from Opposite Sides of the Andes Mountains in Ecuador, with the Description of One New Species. Copeia, 103, 651-663.

Malato, G., Shervette, V.R., Navarrete-Amaya, R., Valdiviezo-Rivera, J., Nugra-Salazar, F., Calle-Delgado, P. ... Aguirre, W.E. (2017). Parallel body shape divergence in the Neotropical fish genus Rhoadsia (Teleostei: Characidae) along elevational gradients of the Western Slopes of the Ecuadorian Andes. PLoS One, 12(6), e0179432.

Moritz, C. (1994). Defining evolutionarily significant units for conservation. Trends in Ecology and Evolution, 9, 373-375.

Myers, N., Mittermeier, R.A., Mittermeier, C.G., da Fonseca, G.A.B., \& Kent, J. (2000). Biodiversity Hotspots for Conservation Priorities. Nature, 403, 853-858.

Nelson, J.S., Grande, T.C., \& Wilson, M.V.H. (2016). Fishes of the World. New Jersey, USA: John Wiley and Sons, Inc. Hoboken.

Provezano, R., \& Barriga, R. (2017). The species of Hemiancistrus (Siluriformes: Loricariidae) from Ecuador. Zootaxa, 4272, 221-235.

Reyes-Corral, W.D., \& Aguirre, W.E. (2019). Effects of temperature and water turbulence on vertebral number and body shape in Astyanax mexicanus (Teleostei: Characidae). PLoS One, 14(7), e0219677.

Rohlf, F.J., \& Marcus, L.F. (1993). A revolution in morphometrics. Trends in Ecology and Evolution, 8, 129-132.

Rohlf, F.J. (2018a). TpsUtil (Version 1.76). Retrieved from http://life.bio.sunysb.edu/morph/ 
Rohlf, F.J. (2018b). TpsDig2 (Version 2.31). Retrieved from http://life.bio.sunysb.edu/morph/

Román-Valencia, C., Ruiz-C., R.I., Taphorn, D.C., \& García-A., C. (2013). Three new species of Bryconamericus (Characiformes, Characidae), with keys for species from Ecuador and a discussion on the validity of the genus Knodus. Animal Biodiversity and Conservation, 36, 123-139.

Sidlauskas, B., Chernoff, B., \& Machado-Allison, A. (2006). Geographic and environmental variation in Bryconops sp. cf. melanurus (Ostariophysi: Characidae) from the Brazilian Pantanal. Ichthyological Research, 53, 24-33.

Strahler, A.N. (1957). Quantitative analysis of watershed geomorphology. Trans Am Geophys Union, 38, 913-920.

Swanne, P.G., Hendry, A.P., \& Reznick, D.N. (2017) Predator-induced Contemporary Evolution, Phenotypic Plasticity, and the Evolution of Reaction Norms in Guppies. Copeia, 105(3), 514-522.

Tan, M., \& Armbruster, J.W. (2012). Cordylancistrus santarosensis (Siluriformes: Loricariidae), a new species with unique snout deplatation from the Río Santa Rosa, Ecuador. Zootaxa, 3243, 52-58.

Thompson, D. (1917). On Growth and Form. Cambridge, UK: Cambridge University Press.

Toro-Ibacache, M.V., Manriquez-Soto, G., \& SuazoGaldames, I. (2010). Morfometría geométrica y el estudio de las formas biológicas: De la morfología descriptiva a la morfología cuantitativa. International Journal of Morphology, 28, 977-990.

Valdiviezo-Rivera, J., Garzón-Santomaro, C., Inclán-Luna, D., Mena-Jaén, J., \& González-Romero, D. (Eds.). (2018). Ecosistemas dulciacuícolas de la provincia de El Oro: Peces y macroinvertebrados acuáticos como indicadores biológicos del páramo al manglar (Publicación Miscelánea No 10). Quito-Ecuador: Serie de Publicaciones Gobierno Autónomo Decentralizado Provincial de El Oro, GADPEO - Instituto Nacional de Biodiversidad, INABIO.

Vannote, R.L., Minshall, G.W., Cummins, K.W., Sedell, J.R., \& Cushing, C.E. (1980). The river continuum concept. Canadian Journal of Fisheries and Aquatic Sciences, 37, 30-137.

Webb, P.W. (1984). Body form, locomotion and foraging in aquatic vertebrates. American Zoologist, 24, 107-120.

West-Eberhard, M.J. (2003). Developmental Plasticity and Evolution. Oxford, UK: Oxford University Press.

Wilson, E.O. (2003). The future of life. New York, USA: Vintage.

Winemiller, K.O., Agostinho, A.A., \& Caramaschi, E.P. (2008). Fish ecology in tropical streams. In D. Dudgeon (Ed.), Tropical stream ecology (pp. 107-146). London, UK: Academic Press.

Zelditch, M.L., Swiderski, D.L., \& Sheets, H.D. (2012). Geometric morphometrics for biologists. A primer. London, UK: Academic Press. 